\title{
Influence of the Rosenzweig functional response on the dynamics of the Leslie-Gower model
}

\author{
EDUARDO GONZALEZ-OLIVARES ${ }^{1}$, Viviana Rivera-Estay ${ }^{1}$, Alejandro Rojas ${ }^{2}$, and \\ Karina VILCHES-PONCE ${ }^{3}$ \\ ${ }^{1}$ Pontificia Universidad Catolica de Valparaiso \\ ${ }^{2}$ Universidad de Chile \\ ${ }^{3}$ Universidad Católica del Maule
}

May 5, 2020

\begin{abstract}
After the well-known classification formulated by Crawford S. Holling in 1959 of the functional responses dependent only of the prey populations, various other have been proposed. In this work a simple Leslie-Gower type predator-prey model is analyzed, incorporating the Rosenzweig functional response described by $\$ \mathrm{~h} \backslash \operatorname{left}(\mathrm{x} \backslash$ right $)=\mathrm{qx} \wedge\{\backslash$ alpha $\} \$$, with $\$ 0<\backslash$ alpha $<1 \$$. This function does not conform to the types proposed by Holling, since is not bounded. Although this functional response is nondifferentiable for $\$ \mathrm{x}=0 \$$, it is proved that the obtained system is Lipschitzian. However, the existence of a separatrix curve $\$ \backslash$ Sigma $\$$ in the phase plane it is proven, which divides the phase plane en two complemntary sectors. According to the relative position of the initial conditions respect to the curve $\$ \backslash$ Sigma $\$$, the trajectories can have differents $\$ \backslash$ omega $\$-\$$ limit $\$$, which can be the equilibrium $\$ \backslash$ left $(0,0 \backslash$ right) $\$$, or else, a positive equilibrium point, or a limit cycle or a heteroclinic curve. These properties show the great difference of this model with the original and well-known Leslie-Gower model (when $\$ \backslash$ alpha $=1 \$$ ), since this last has only a unique positive equilibrium, which is globally asymptotically stable. Then, it can concluded that i) a small change in the mathematical expression for the functional response, it produces a strong change on the dynamics of model. ii) \a slightest deviation in the initial population sizes, respect to the curve $\$ \backslash$ Sigma $\$$, it can signify the coexistence of populations or the extinction of both. Numerical simulations are given to endorse our analytical results.
\end{abstract}

\section{Hosted file}

03LESLIENONDIFFSPEC.pdf available at https://authorea.com/users/303582/articles/433787influence-of-the-rosenzweig-functional-response-on-the-dynamics-of-the-leslie-gowermodel 
figures/fig1/fig1-eps-converted-to.pdf 
figures/sepx/sepx-eps-converted-to.pdf 
figures/Sim1/Sim1-eps-converted-to.pdf 
figures/Sim2/Sim2-eps-converted-to.pdf 
figures/Sim3/Sim3-eps-converted-to.pdf 
figures/Sim4/Sim4-eps-converted-to.pdf 
figures/Sim5/Sim5-eps-converted-to.pdf

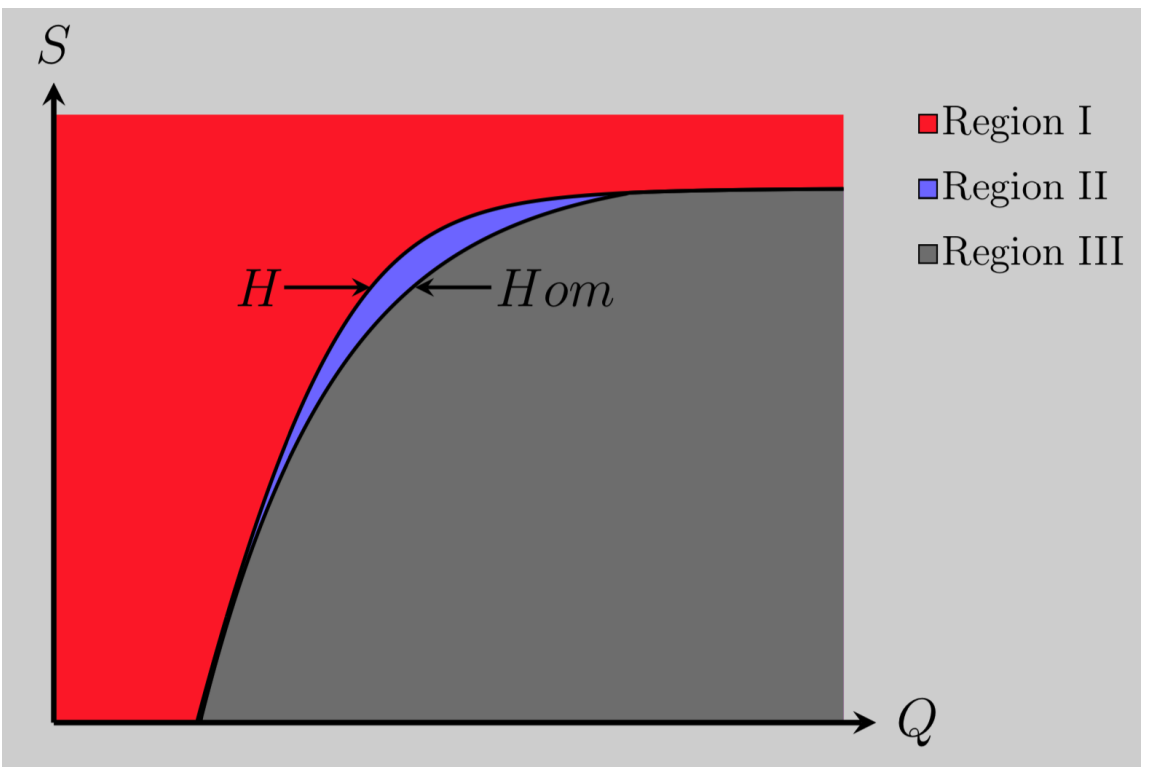



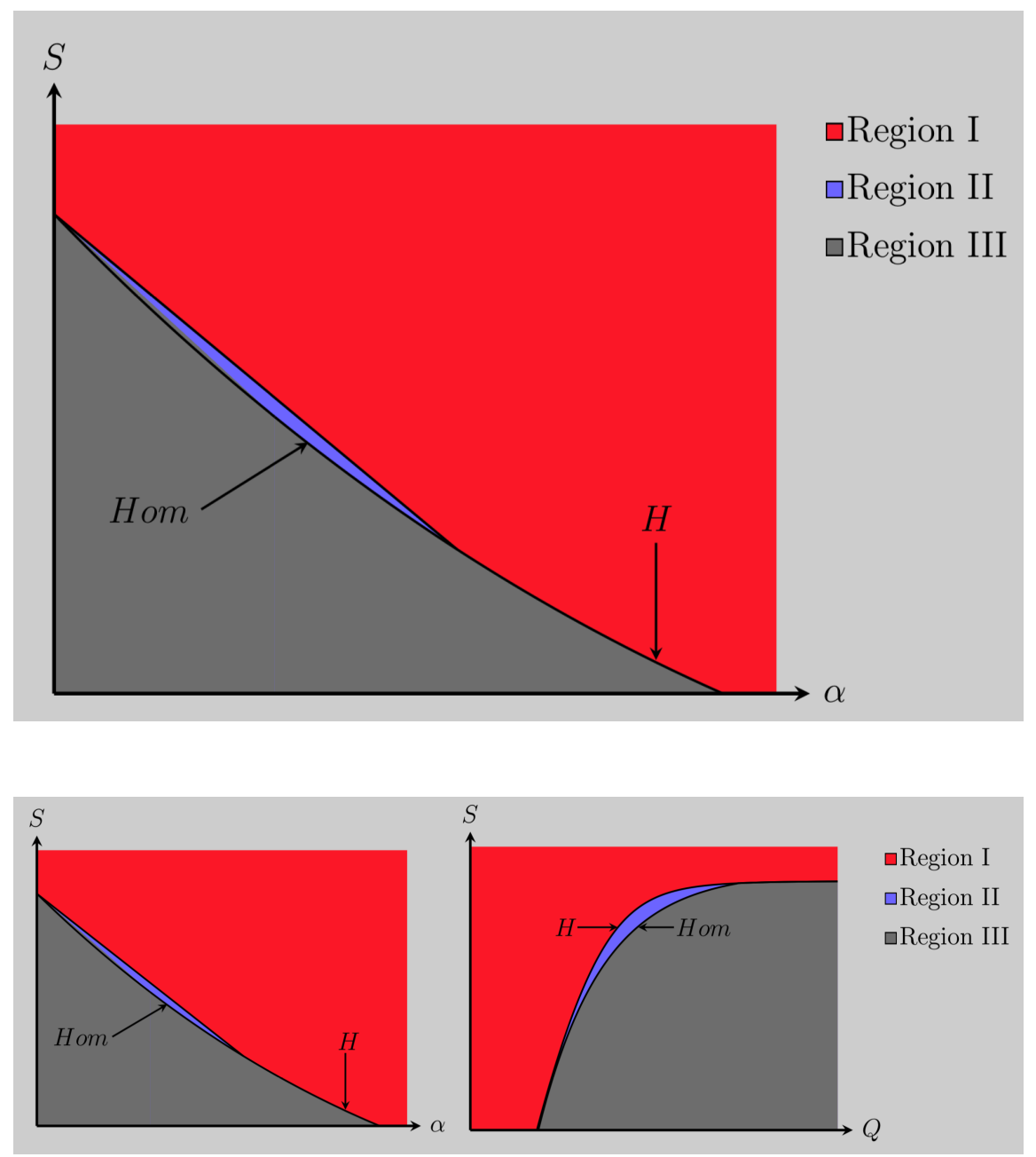